\title{
Numerical Simulation and Experiment Study of Double Rails Propelle Type Crossing Frame
}

\author{
YJ Xia ${ }^{1, a}$, TG Yu ${ }^{2, b}$,YS Hu ${ }^{1, c}$ and SL Cai ${ }^{1, d}$ \\ ${ }^{1}$ China Electric Power Research Institute, State Grid of China, 100192 \\ ${ }^{2}$ Shanghai Power Transmission and Transformation Engineering Company, State Grid of China, \\ 200235 \\ axiayj@epri.sgcc.com.cn, byutiangang2007@163.com, chansonncut@163.com, ${ }^{\mathrm{d}}$ caisonglin@epri.s \\ gcc.com.cn
}

Keywords: Crossing frame; Static load; Test; Numerical simulation; Construction technology.

\begin{abstract}
Based on stringing construction of the conductor in power transmission engineering construction, Shanghai Power Transmission and Transformation Engineering Company designed the double rails propelle type crossing frame, which was used to protect the important crossed objects. Before the crossing frame is utilized, the numerical simulation study and the load test study are carried out. This paper describes the study methods for the numerical simulation study and the load test of the double rails propelle type crossing frame, the testing conditions of the crossing frame are determined through analyzing the basic operation conditions of the crossing frame, and the numerical simulation study of the testing operation conditions of the crossing frame is carried out. Safety and stability of the crossing frame are inspected through comparison between the test result and the simulation result, which greatly ensures safety of the crossing frame during engineering application.
\end{abstract}

\section{Introduction}

Following development of the grid construction in great scale and complete economic and social development in our country, the crossing of the power line under construction, the power line in service, the high speed railway and the high speed road etc special crossing conditions are increased quickly, the crossing construction of the tension stringing is more and more regular. Simultaneously, the country focuses on safety production works more and more, requirements are improved gradually, and danger point during the crossing construction is more and safety risk is great, in particularly that safety and reliability of the construction of the high voltage crossing and super-high voltage live line, the high speed railway have significant meaning on smooth implementation of the grid construction and safety operation of the high speed railway. In case safety production accident occurs, it will cause great economic loss and social influence.

In order to protect the crossed object during crossing construction of the tension stringing, the way of setting up the crossing frame is regularly applied at present. In order to improve technical level of the crossing construction, power-off time during crossing shall be shortened as possible, which can practically guarantee safety of the crossing construction. Shanghai Power Transmission and Transformation Engineering Company of State Grip Corporate designs one metal lattice type crossing frame for stringing construction of the railway crossing, i.e., the double rails propelle type crossing frame shown as figure 1 , which has maximum span of $60 \mathrm{~m}$ and enclosed network width of $8 \mathrm{~m}$.

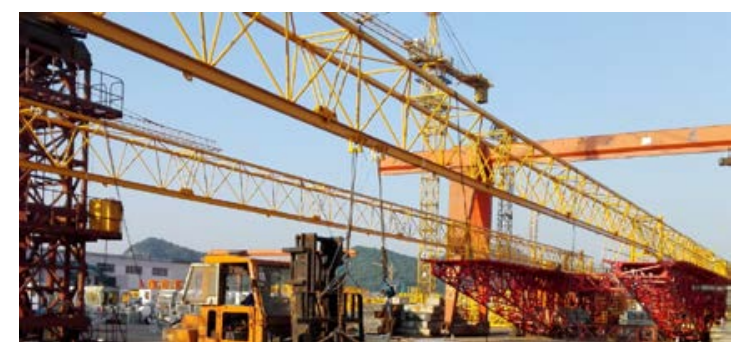

Fig. 1 Hard enclosed network system of double rails propelle type crossing frame 
For design and test of the crossing frame and the crossing construction engineering process, several power industrial standards in our country and enterprise standards of State Grid Corporate have made regulations, but there are still many problems:

(1) There are great technical gaps in theoretical analysis technology of the crossing frame: actual impact influence of the conductor under accidental operation condition of the actual crossing frame isn't considered in the calculation analysis method, safety and economy for design of the crossing frame can't be guaranteed.

(2) There are great technical gaps in the test and detection technology of the crossing frame: actual bearing capability of the crossing frame can't be estimated. A large quantity of the new brand crossing frames which are innovatively designed by the power transmission and transformation construction unit are extensively applied during crossing construction at present, and safety performance of the crossing frame can't be mastered.

(3) Standardization works of the crossing frame shall be further improved. In order to validate whether safety and stability of the double rails propelle type crossing frame meet with the design and engineering application requirements, simulation of the crossing frame before putting into operation is carried out, and the load test of the crossing frame is also carried out. The numerical simulation result and the test results are finally compared and analyzed, and then the reasonable evaluation and theoretical analysis study on safety and stability of the crossing frame are carried out.

\section{Composition of Double Rails Propelle Type Crossing Frame}

The double rails propelle type crossing frame mainly consists of the positioning platform, the weight bearing platform, the weight bearing gantry, the hard enclosed network rod, the crossing beam and the butt joint platform etc. Its three dimension schematic figure is shown as fig. 2.

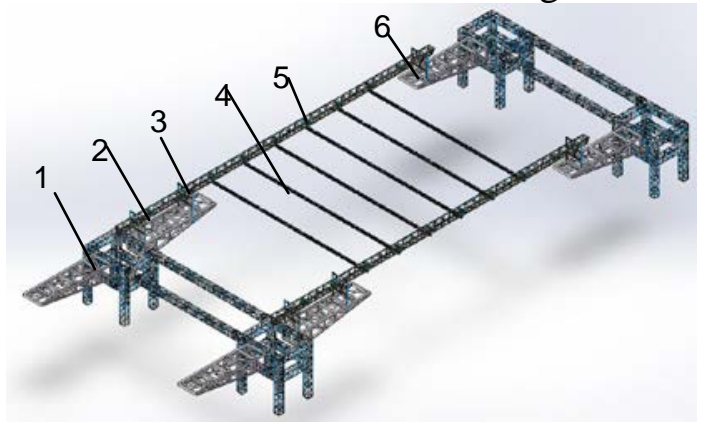

1-Positioning platform; 2- Weight bearing platform; 3- Weight bearing gantry; 4- Hard enclosed network rod; 5- Crossing beam; 6- Butt-joint platform.

Fig. 2 Schematic figure of double rails propelle type crossing frame

The crossing frame standard joints of the crossing frame are connected on the positioning platform, which are fixed on the weight bearing gantry and the positioning gantry, the crossing beams of the crossing frame are jacked and pushed joint by joint through the hydraulic push device, which can realize installation of the crossing beam of the crossing frame. The trolley is installed on the crossing beam of the crossing frame, the hard enclosed network rod is suspended at lower, the hard enclosed network rod is towed to working position by the traction device, the nylon enclosed screen is installed between the hard enclosed network rod, and then realize installation of the crossing frame.

\section{Analysis of Test Condition}

Installation and application process for analyzing of the crossing frame consists of four basic operation conditions according to its load conditions, i.e., installation operation condition, safety stringing operation condition, accident simulation operation condition and gale operation condition. The gale operation condition means the crossing construction has been stopped when wind force reaches six class and above, load at accidental operation condition shall not be considered for the crossing frame, it only bears dead weight wind load. Because wind load can't be simulated, this condition isn't considered during design. When wind force is great, prevention measures to 
temporarily remove a part of the member s of the crossing frame shall be applied, the gale operation condition test shall not be carried out. Therefore,the test operation conditions consist of 3 types, including: installation operation condition, safety stringing operation condition and accidental simulation operation condition (these three test operation conditions are tested under breeze or no wind conditions, wind load influence is also not considered).

(1) Installation operation condition

When the end of the crossing beam of the enclosed network is pushed to position of the lifting platform during installation process of the crossing frame, the crossing beam of the enclosed network is placed at maximum suspension arm status, it will bear large bending moment of dead weight at section of the weight bearing gantry, the upper and lower main chord rods will bear large drawing force and compression force respectively, the end of its suspension arm is maximum deformation position, it is maximum forcing operation condition during its installation process, shown as fig.3.

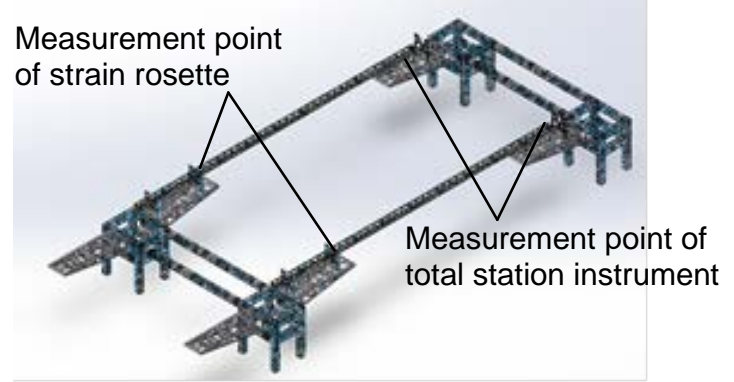

Fig.3 Schematic figure of installation operation condition

(2) Safety stringing operation condition

When the enclosed network crossing beam of the crossing frame is installed on position, the aluminium alloy rod of the enclosed network is towed to position by the trolley. After the top enclosed, network is suspended and installed, installation of all components of the crossing frame are installed, the crossing construction conditions are available. Now accidental operation condition load isn't necessary to be considered for the crossing frame, it only bears dead weight, the middle crossing position of its enclosed network crossing beam is its maximum forcing position and maximum deformation position, shown as fig. 4 .

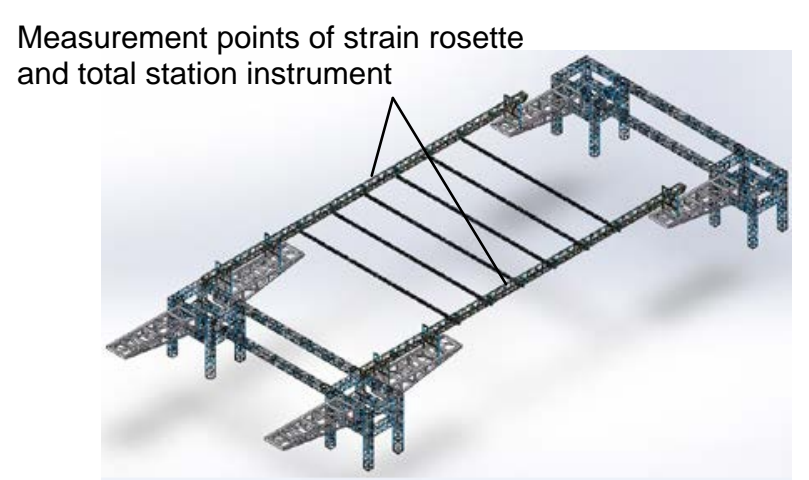

Fig. 4 Schematic figure of safety stringing operation condition

(3) Simulation accidental operation condition

The crossing frame will bear impact action of conductor deflection and conductor breakage load under accidental operation condition. Because static design and static test are carried out, the simulation accidental operation condition test through imposing static design load is carried out. Now the middle crossing position of the single side enclosed network crossing beam of the crossing beam will bear static simulation accidental load, the middle crossing position of the forced single side enclosed network crossing beam is maximum forcing position and maximum deformation position, shown as fig. 5. 


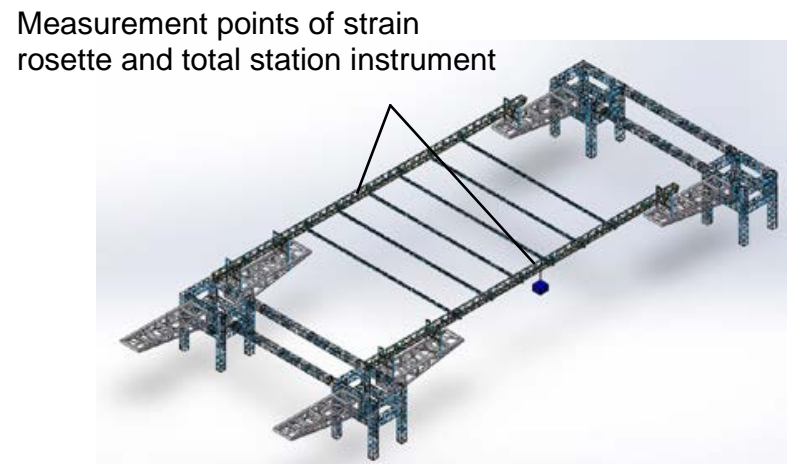

Fig.5 Schematic figure of simulation accidental operation condition

Static simulation accidental load (i.e., design load) of the crossing frame has been discussed and determined by Infrastructure Department of State Grid Corporate and Shanghai Power Company of State Grip Corporate through organizing the meeting, which is 2 tons. Position that the conductor impacts on the crossing frame isn't its right middle position at accidental operation condition, there is deflection, static simulation load is completely imposed on the crossing beam of the crossing frame at one side during design, limit operation condition is considered.

\section{Numerical Simulation Analysis}

Establish the numerical simulation analysis model of the crossing frame according to the actual design parameters and the materials of the crossing frame. Stress and deformation displacement value of every maximum forcing point at every operation condition of the crossing frame are calculated through numerical simulation analysis, and the theoretical evaluation of safety of the crossing frame is also carried out.

(1) Installation operation condition

Establish the numerical simulation model at installation operation condition according to status under installation operation condition of the crossing frame, shown as fig. 6 .

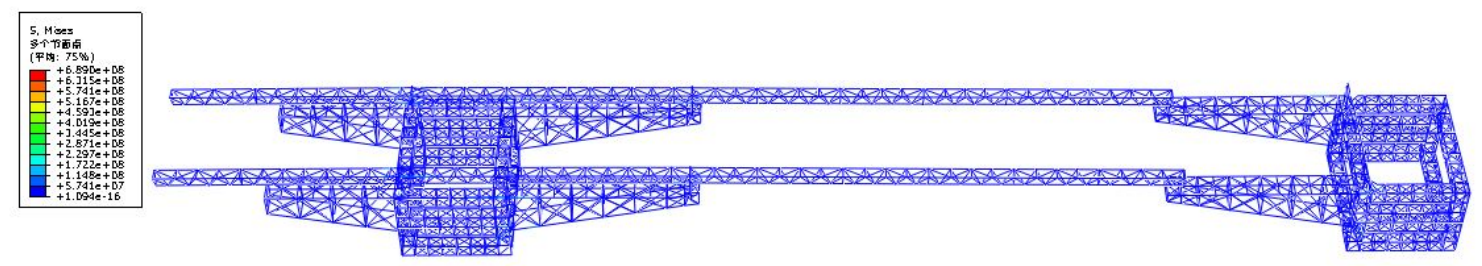

Fig.6 Stress cloud figure at installation operation condition

Maximum stress of the crossing beam at position of the weight bearing gantry is $10.1 \mathrm{MPa}$, maximum strain is $43 \mu \varepsilon$, maximum displacement at end of the suspension arm is $52 \mathrm{~mm}$.

(2) Safety stringing operation condition

Through establishment of the model, the crossing frame only bears dead weight at safety stringing operation condition, its forcing cloud figure can be obtained, shown as fig.7.

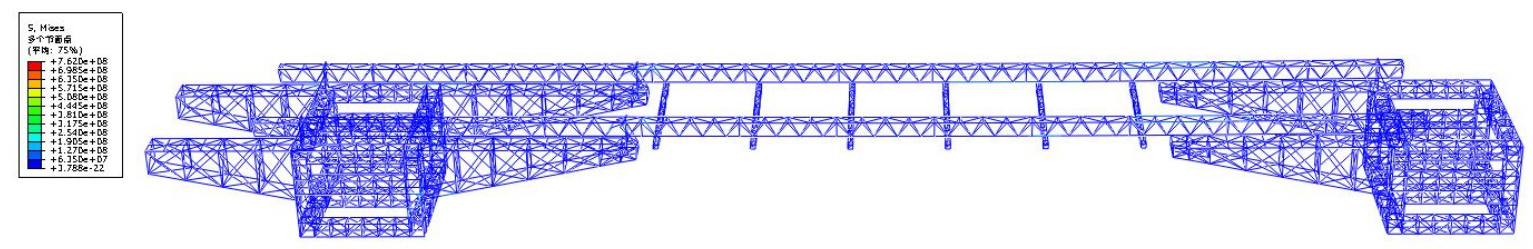

Fig.7 Stress cloud figure at safety stringing operation condition

Maximum stress is $8.4 \mathrm{MPa}$, maximum strain is $35 \mu \varepsilon$, maximum displacement is $33 \mathrm{~mm}$, the position is located at the sectional steel beam in the middle position of the crossing beam, its maximum stress value is slightly less than maximum stress value at installation operation condition. 
(3) Simulation accidental operation condition

Middle crossing of the crossing beam of the enclosed network at single side only bears load of 2 tons under simulation accidental operation condition, its stress cloud figure is shown as fig. 8 .
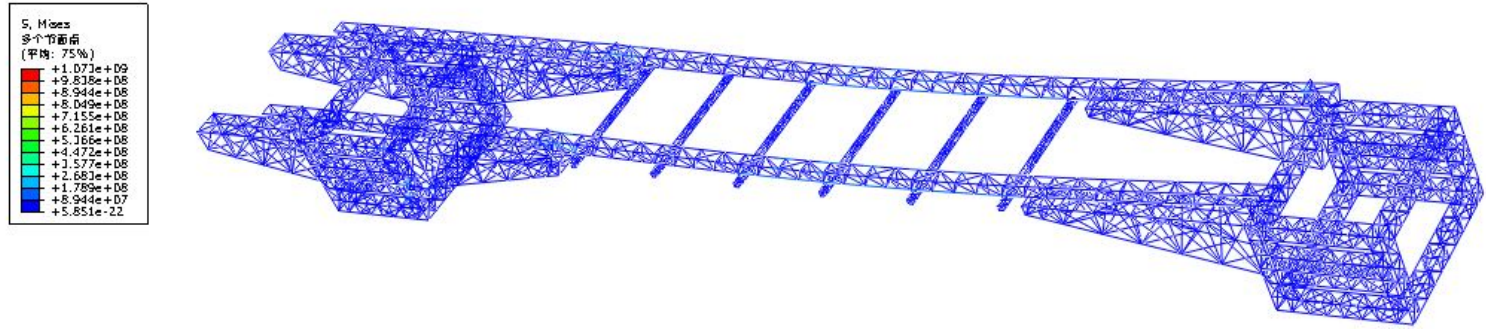

Fig.8 Stress cloud figure at simulation accidental operation condition

The maximum middle crossing stress of the crossing beam which is obtained through calculation analysis is $43.1 \mathrm{MPa}$, maximum strain is $152 \mu \varepsilon$, and maximum displacement is $93 \mathrm{~mm}$, the position is located at the sectional steel beam in the middle of the crossing beam, its maximum stress is only slightly less than maximum stress value under installation operation condition.

\section{Experiment}

The maximum stress and maximum displacement position of the crossing frame at every operation condition during test operation condition analysis are determined, the point is arranged through adhering the stain rosette, the main strain at the measurement point is calculated and then the equivalent stress is obtained, deformation displacement of the crossing frame is measured by the total station instrument, arrangement of every measurement point is shown as figure.9.
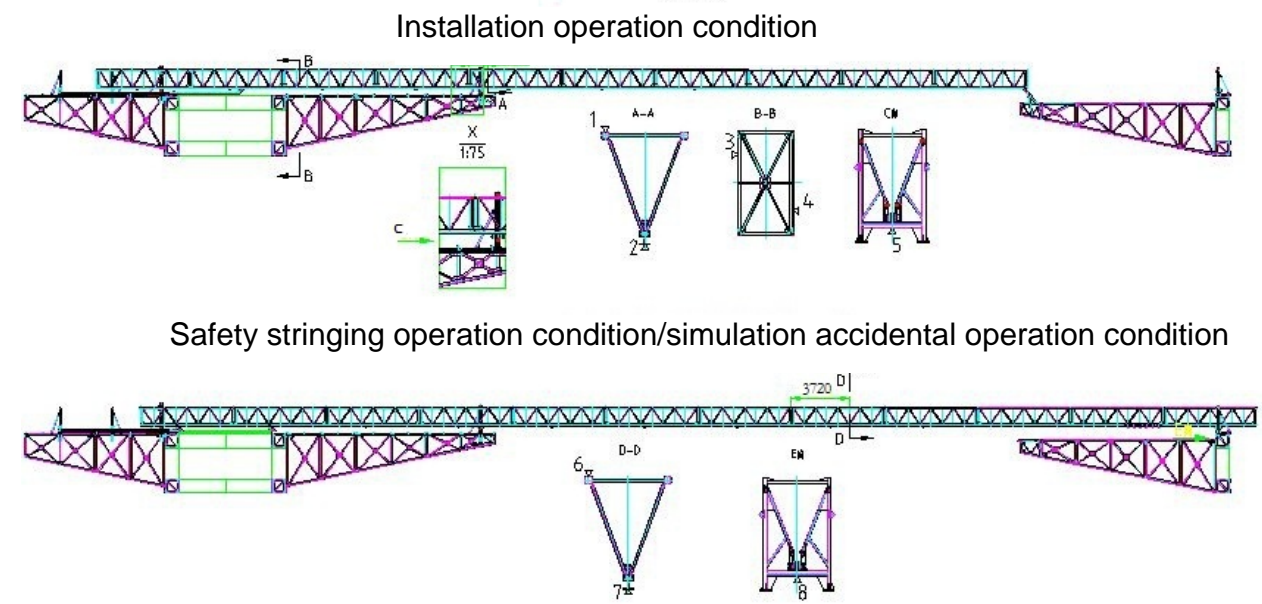

Fig.9 Arrangement figure of measurement point of load test

Strain value at every measurement point is measured by the dynamic strain instrument and torsion at every measurement point is measured by the total station instrument, which are used forcalculation analysis.

Analyzing the strain status of the plane, main strain is calculated according to Eq.1[1]:

$$
\left\{\begin{array}{l}
\varepsilon_{\max } \\
\varepsilon_{\min }
\end{array}=\frac{\varepsilon_{0^{0}}+\varepsilon_{90^{0}}}{2} \pm \sqrt{\left(\frac{\varepsilon_{0^{0}}+\varepsilon_{90^{0}}}{2}\right)^{2}+\left(\frac{\varepsilon_{0^{0}}+\varepsilon_{90^{0}}-2 \varepsilon_{45^{0}}}{2}\right)^{2}}\right.
$$

Where $\varepsilon_{\max }$ and $\varepsilon_{\min }$ are main strain, ${ }^{\varepsilon_{0^{0}}}$ is strain at direction of 0 degree, ${ }^{4^{\circ}}$ is strain at direction of 45 degree, ${ }^{\varepsilon_{90}}$ is strain at direction of 90 degree.

Main stress shall be calculated according to Eq.2[1]: 


$$
\left\{\begin{array}{l}
\sigma_{1}=\frac{E}{1-\mu^{2}}\left(\varepsilon_{\max }+\mu \varepsilon_{\min }\right) \\
\sigma_{2}=\frac{E}{1-\mu^{2}}\left(\varepsilon_{\min }+\mu \varepsilon_{\max }\right)
\end{array}\right.
$$

Where ${ }^{\sigma_{1}}$ and ${ }^{\sigma_{2}}$ are main stress, $E$ is elastic module, ${ }^{\mu}$ is poisson ratio, ${ }^{\varepsilon_{\max }}$ and ${ }^{\varepsilon_{\min }}$ are main strain. Calculate equivalent stress of the strain rosette according to main stress at two directions of the plane, equivalent stress shall be calculated according to Eq.3[1]:

$$
\sigma_{E q}=\sqrt{\frac{\left(\sigma_{1}-\sigma_{2}\right)^{2}+\left(\sigma_{1}\right)^{2}+\left(\sigma_{2}\right)^{2}}{2}}
$$

Where ${ }^{\sigma_{E q}}$ is equivalent main stress, ${ }^{\sigma_{1}}$ and ${ }^{\sigma_{2}}$ are main stress.

Equivalent stress value at every measurement point is calculated according to above calculation method.Through data analysis,Equivalent maximum stress of beam is 174MPa. Maximum deflection of beam is $288 \mathrm{~mm}$. The result meets the requirements.

\section{Summary}

After load test and numerical simulation are carried out by the double rails propelle type crossing frame, the test result is compared and analyzed with the simulation result, and danger section of load test of the crossing frame is determined, and the following conclusions are obtained:

(1)Test result is very close to simulation value, it testifies reasonability of simulation model establishment.

(2)Carry out reasonable evaluation on safety of design of the crossing frame through test result, the crossing frame meets with application requirement of operation condition.

(3)Add position of the arranged point, further analyze forcing condition of other structure of the crossing frame and compare with the simulation value.

\section{Acknowledgement}

This research was financially supported by the Study on Design and Construction Technology of Transmission Line Lattice Frame Project of China State Grid Corp.

\section{References}

[1] HW,Liu: Mechanics of materials ,Higher Education Press Fifth Edition (2003).

[2] L P,Bai: Discussion on dynamic-static load test of highway bridge structure (2013), p. 150.

[3] R M,Li, etc.: Research of structure test based on the large load/deformation turbine blade(2013), p.53.

[4] YC,Z:Static Load Study of Rigid Frame Arch Bridge.(2012).

[5] R,Lu:The application of cover excavation method in loess tunnel construction(2013). 